MedienPädagogik

www. medienpaed.com
Zeitschrift für

Theorie und Praxis der Medienbildung

ISSN 1424-3636

Themenheft Nr. 19: Mobile Learning in Widening Contexts: Concepts and Cases

\title{
Context in a Wider Context
}

John Traxler

\begin{abstract}
This paper attempts to review and reconsider the role of context in mobile learning and starts by outlining definitions of context-aware mobile learning as the technologies have become more mature, more robust and more widely available and as the notion of context has become progressively richer.

The future role of context-aware mobile learning is considered within the context of the future of mobile learning as it moves from the challenges and opportunities of pedagogy and technology to the challenges and opportunities of policy, scale, sustainability, equity and engagement with augmented reality, ‘blended learning),

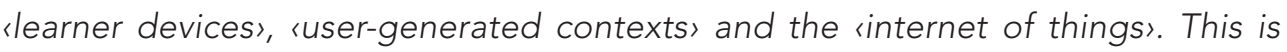
essentially a perspective on mobile learning, and other forms of technologyenhanced learning (TEL), where educators and their institutions set the agenda and manage change.

There are, however, other perspectives on context. The increasing availability and use of smart-phones and other personal mobile devices with similar powerful functionality means that the experience of context for many people, in the form of personalized or location-based services, is an increasingly social and informal experience, rather than a specialist or educational experience.

This is part of the transformative impact of mobility and connectedness on our societies brought about by these universal, ubiquitous and pervasive technologies. This paper contributes a revised understanding of context in the wider context (sic) of the transformations taking place in our societies. These are subtle but pervasive transformations of jobs, work and the economy, of our sense of time, space and place, of knowing and learning, and of community and identity. This

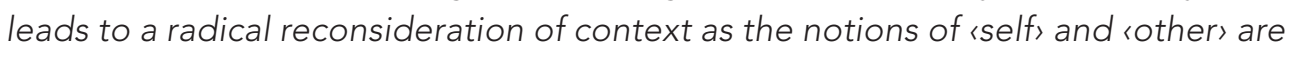
transformed.
\end{abstract}

\section{Introduction}

The structure of the paper is as follows: we start by considering context-aware learning as a specific focus within mobile learning research, itself probably a vanguard within researchers engaged in technology-enhanced learning, and how its development has led to progressively richer conceptions of context. The second half of the paper looks at the impact of wider mobility and connectedness, specifically at how the conventional conceptualisation of context creates a 
dichotomy between «self〉, the user or learner, and «other〉, the environment, the surroundings.

\section{Context in Mobile Learning}

We must start with a brief review of the notion of «context) and the role it has played in mobile learning. It has been as one of the defining contributions of mobile learning to the wider field of technology-enhanced learning, exploiting the personal and portable nature of the devices and their capacity to sense some aspects of their context, initially their location and trajectory. Context has been defined and classified in a variety of different ways. A working definition might be, "the formal or informal setting in which a situation occurs; it can include many aspects or dimensions, such as environment, social activity, goals or tasks of groups and individuals; time (year/month/day).» (Brown 2010:7) or "any information that can be used to characterize the situation of an entity, where an entity can be a person, place, or physical or computational object» and thus, context-awareness or context-aware computing is "the use of context to provide task-relevant information and/or services to a user» (Dey \& Abowd, 1999:1), "typically the location, identity and state of people, groups, and computational and physical objects». The same source looks at context from different perspectives. It makes the distinction between, on the one hand, those contexts that relate to the user's environment, including attributes such as their emotional state, focus of attention, social and informational state, and, on the other hand, those contexts relating to the application's environment, surroundings, settings or states, or the current environment as a whole. Some attributes are common to both types of context including location, time of day, season, temperature, identities of people and objects around the user and changes to these identities. At least two other classifications of contexts have been proposed. The first (Schilit et al., 1994; Chen \& Kotz, 2000), defines four categories of contexts:

- Computing context including network connectivity, communication costs and bandwidth, nearby resources such as printers, displays and workstations, though this however becomes progressively less significant as the factors concerned become more stable, uniform, transparent and capable.

- Physical context including lighting, noise levels, traffic conditions and temperature though these too may also become less significant as devices become more impervious to many of these aspects of physical context.

- User context including the user profile, location, people nearby and current social situation. Increasingly devices might give orientation and might sense or even recognise objects in their vicinity so this aspect continues to become richer and the internet of things (Siorpaes et al., 2006) increasingly draws the physical world and its objects into the virtual world.

- Time context including obviously time of day, week, month and season of year. 
The second classification (Schmidt et al., 1998), contains two categories from the two different perspectives.

- Human factors

- User, their personal habits, mental state, etc.

- Social environment, namely the proximity of other people, social relations, collaboration

- Task, any goal directed activities or more general objectives

- Physical environment

- Location

- Infrastructure, the interactive and computing environment

- Conditions such as the level of noise, brightness etc.

There were also attempts to classify contexts specifically in relation to learning; one source, for example (Wang, 2004) breaks learning contexts into six dimensions: identity, spatio-temporal, facility, activity, learner and community. Identity characterises the unique learner, spatio-temporal is the time and location aspect of the learning process, facility is the type of mobile device being used, activity categorises the learning activity taking place, such as individual or collaborative, the learner dimension describes characteristics such as 〈learning style» and knowledge level and the community aspect describes the social interactions between participants.

These distinctions are typical of attempts to define and delineate precise aspects of context. More recently, however, the distinction between the learner or user and their context or environment has been eroded by the notion of suser-generated contexts' (Cook, 2010), a concept named to emphasise the role of learners themselves in shaping their own context, "the context within which communication takes place is augmented by users to suit the needs of the individual and/or the conversational community» (Cook et al., 2010:4).

Alongside these developments, a more philosophical debate takes place, characterising our description so far as largely only one perspective of two (Dourish, 2004: 20). It is «a technical notion, one that offers system developers new ways to conceptualise human action and the relationship between that action and computational systems to support it». The other view draws «analytic attention to certain aspects of social settings» and argues that the earlier position was essentially positivist and that there is an alternative viewpoint, one of phenomenology. It leads to a different view of context. Rather than context as information, it is «a relational property that holds between objects or activities» and thus signifies relevance; rather than delineated and defined in advance, context is defined dynamically; rather than stable, context is local to each occasion of activity or action; rather than context and content being two separable entities, "context arises from the activity. 
Context isn't just «there», but is actively produced, maintained and enacted in the course of the activity at hand» (Dourish, 2004:22).

Context can thus also be proximity and relevance as we have seen, and as difference and change; it can be seen as a description of that which differentiates, what is different in what is near or recent and what is further or earlier in relation to the subject. Originally, these factors related to physical or geometric distance but clearly there are other aspects, perhaps social proximity or pedagogic proximity. An early definition of context gets near to these other ideas of proximity, defining it as "location and the identity of nearby people and objects» (Schilit \& Theimer, 1994) and argue for social dimensions, saying: "Context encompasses more than just the user's location, because other things of interest are also mobile and changing. Context includes lighting, noise level, network connectivity, communication costs, communication bandwidth and even [our emphasis] the social situation, e.g., whether you are with your manager or with a co-worker.»

Perhaps the last word for the moment should be, "Context» is a slippery notion. Perhaps appropriately, it is a concept that keeps to the periphery, and slips away when one attempts to define it» (Dourish, 2004:29).

\section{How Things Have Changed}

These developments in the ideas and practice of context-aware mobile learning came, however, out of a particular historical and social milieu. They were embedded deeply within the mobile learning research community and seen as a major development and contribution to the wider field of technology enhanced learning. In the early days of mobile learning, perhaps the first six or seven years of this century, these developments helped place the mobile learning research community amongst the vanguard of technology-enhanced learning in an era when most people's, most learners' access to powerful and expensive educational technologies was through educational institutions. This was due to their relative cost, bulk and scarcity; it was mainly networked desktop computers running dedicated educational software integrated into wider institutional software systems, such as library software and student enrolment software. It was and partly still is the corollary of an institutional culture in which research and development in technology-enhanced learning formed part of a «research economys, where institutional research teams bid for project funding, conduct the research, usually in-house, small-scale and by definition fixed-term, publish the results and move onto the next funding opportunity. It was also part of an expectation that the institution would procure, provide, install and control any subsequent deployment of learning technology and it was part of a related expectation that the institution would promulgate technical and operational standards for learning technology in order to ensure equity, accessibility, uniformity and cost-effectiveness. 
Now, for reasons of cost and sustainability, the focus has moved to «learner devices», those owned by learners (Traxler, 2010a) and with it the locus of agency and control has moved from the institution to the learner. This is challenging and complex and leaves much to be negotiated; but if the professionals within institutions can accept the challenges then scale and sustainability become distinct possibilities for mobile learning in general including context-aware mobile learning. Learner devices, actually just devices typical of the current retail domain, offer locationawareness and often augmented reality along with much other sophisticated functionality. However attractive this may sound, the challenges include equity, standards, quality assurance, infrastructure, security and embedding and blending with institutional «blended learnings.

Much of the work so far could be seen in retrospect as an aspect of a web1.0 ideology, where the user is merely the reader, consumer or recipient. There was, however, some work with a more web2.0 perspective, where users were writers, producers and contributors. This was educational, recreational, reflective, expressive and creative.

One limitation of many early projects was that their temporal and sometimes spatial aspects were bounded or episodic; not only were the projects themselves fixed-term and small-scale, but the experience of the individual learner or user was limited to a short time, the length of their visit, and perhaps to a particular physical location such as a museum or heritage site. Outside these bounds, the experience was either exhausted or curtailed; and the predominant aspects of context were usually just spatial and temporal. The increased technical capacity, functionality and connectivity, especially indoors and in metropolitan areas, however, offer a financially sustainable way to move beyond the merely local, anonymous and episodic experiences of much of this earlier informal context-aware mobile learning and offer a much richer experience as well. It is possible to imagine in the near future, perhaps linked museums for example the Ironbridge Gorge Museums in Shropshire or the South Kensington museums in London or national organisations with heritage sites such as English Heritage or the National Trust where successive or repeat visits of groups or individual using their own devices build a cumulatively richer and perhaps more collaborative experience.

\section{The Mobility and Connection of Society}

The last three or four years have seen a rapid change in the ownership of powerful digital technologies for learning. As we said, this was previously predominantly uniform networked desktop computers in educational institutions; now it is highly functional but diverse and rapidly changing personal mobile phones across the vast majority of our society, learners and others alike. 
Consequently, we now cross from a technical or reformist account of context to a radical or social account and a shift of context-aware mobile learning from a component of mobile learning to the educational component of context-aware services and experiences.

This is having dramatic, though maybe unnoticed, implications for the role, direction and significance of the mobile learning research community, as we shall see. It has implications for the relationships between learners and their educational institutions. It also has a profound, pervasive but subtle impact on work, jobs, businesses and the economy; on perceptions of time, space and place; on the individual, their identity and the nature of communities; on knowledge, knowing, understanding and learning and on consequently on a changed meaning for «context〉 and its role in education. Our underlying content is that earlier work on context-aware mobile learning was predicated on specific fixed ideas about «self〉 and (other), as articulated within institutions, and that the impact of wide social mobility and connectedness in the way we outline have significantly transformed these ideas.

\section{$5 \quad$ Time and Space}

We will start by looking briefly at time, space and place and their implications for «context». We draw on what we have said elsewhere (Traxler, 2010b).

Firstly, interacting with a desktop computer and thus entering cyberspace takes place in a bubble, in dedicated times and places where the user has their back to the world for a substantial and probably premeditated episode. Interacting with a mobile is different and woven into all the times and places of users' lives. Whereas the desktop computer imposes quite a rigid and separate set of contexts on a user or learner - they are either learning or they are doing something else equally specific -, mobiles produce or enforce a more fragmentary and transient movement between multiple user-contexts. One consequence of shift of cyberspace from desktop to mobile is that real and virtual spaces and the contexts that they represent become interwoven ... the user works now in overlapping and fragmentary contexts, where other roles or contexts can easily intrude. Users are no longer dedicated learners nor are they stable contexts.

Mobile technologies erode ideas of physical time as the common temporal context and the temporal context «...can instead be socially negotiated» (Sørensen et al., 2002:3) alongside the "softening of schedules» (Ling, 2004:73) afforded by mobile devices. Nyíri (2006:301) says: «with the mobile phone, time has become personalized». Or perhaps, «... this means the replacement one time by a series of overlapping times» (Cooper, 2001:25) or overlapping temporal contexts. Nowadays, "One can be interrupted or interrupt friends and colleagues at any time. Individuals live in the phonespace - they can never let it go, because it is their primary link 
to the temporally, spatially fragmented network of friends and colleagues they have constructed for themselves. It has become their new umbilical cord, pulling the information society's digital infrastructure into their very bodies. In fact, as technical evangelists at Nokia pondered, mobile communications could eventually evolve into an activity indistinguishable from telepathy.» (Townsend, 2001:70). Mobile Times, a recent Intel project (http://papr.intel-research.net/projects.htm \#time), talks about time becoming (plastic〉 saying: «The experience of «plastic times frames modern life. It is an experience that is highly interruptible, shrinking and expanding around immediate concerns, and interleaving through multiple activities». Elsewhere Intel researchers say: "Conflicts arise not when people have more to do, but increasingly diverse things to switch between, creating the need to «shift gears ' frequently». This sounds to a computer scientist like the overheads associated with «context-switching) (sic).

Agar (2003: 4) makes a direct comparison between the mobile phone and wristwatch, in terms of intimacy and ownership. He also, however, makes a contrast, a direct contrast in terms of personal freedom: «while it might have felt like liberation from tradition, the owner was caught anew in a more modern rationality, for, despite the fact that the pocket watch gave the owner personal access to exact time, accuracy depended on being part of a system». In fact, it made the owner part of a system, part of a stable universal temporal context. Wristwatches are handcuffs, keeping the wearer in only one temporal context at a time (sic). Time zones, another temporal artefact of the Industrial Revolution, devised in the aftermath of the new national railway networks, have a similar effect of creating a large-scale unified and monolithic temporal context.

Now, of course, personal mobile connectedness (and the mobility of the car) erodes these too; international travellers are no longer locked into their local time zone, their own local temporal context. Mobile phones mean they are also tethered to family temporal contexts back home and to the rhythms of their office and colleagues back at base or across a range of global offices rather than exclusively to their own physical context, location and contacts.

Staid (2008:157), in talking about «the phone as mobile log», reminds us that mobiles are "a kind of life diary that saves experiences, memories, thoughts, or moments in a visual and textual form. The sim card in your phone could be seen to contain the story of your life (at least at the present time): not just text messages, photos and videos, but also chosen or given tokens such as icons, ring tones, music lists; and the diary, address book, alarm clock all save and display the experiences and activities of the user as they have been mediated and captured by the mobile.» This second-by-second account of our lives is another way in which mobiles transform our sense of time passing, populating our personal user-generated temporal 
context with an unprecedented level of detail, making it more finely-grained whilst plastic and fractured.

Furthermore, the increase of rolling news, off-air recording and domestic video-ondemand means that TV schedules no longer provide a synchronous and collective context that bound informal groups together in the way they used to a generation ago. Students can no longer all discuss last night's programmes when they arrive at university, each relating to a common temporal and social context. Ling (2008:62) makes a similar point, citing the lost rituals of watching national TV news in Norway saying: «In this way the TV, and the particular news program, provided a kind of ontological security». At the same time, news is becoming more local, the news of your online community rather than global news (Bilton, 2010), altering the shape of the informational context of the user, reconfiguring the informational, historical and social contexts of users as the old landmarks are re-aligned, removed or replaced.

\section{Place, Space and Presence}

Mobile devices are accelerating the erosion of physical place as the predominant aspect of the spatial context started by other networked digital technologies. It is being diluted by «absent presence» (Gergen, 1996), the phenomenon of physically social co-located groups all connected online elsewhere and by «simultaneity of place» (International Telecommunications Union, 2004:20, paraphrasing Plant, 2000) created by mobile phones, that is one physical space and multiple mobile virtual spaces of conversational interaction instead of a solid stable spatial context.

Fortunati (2002:515) relates absent presence to a discussion of personal and public spaces, suggesting a preference for the known and private that forces us away from the physical. "[Y]ou have the possibility of choosing between the public space of streets, stations, means of transport and the private space of interpersonal relationships, between chance socialness which may develop with those who happen to be passing by, and chosen socialness (e. g. with the friends you decide to call on your mobile phone). And it is obvious that the choice always falls on the second, if only because it represents the encroaching 〈new〉.»

She continues by eliding absent presence with motion and then discussing the value and valorisation of space: "This phenomenon is evident in means of transport. Compartment conversation, a typical communicative mode in which nothing very important is said, is increasingly often silenced by selected but artificial conversations (that is, by the mobile). The individual is in one place, as a physical presence, but virtually, as an immaterial presence, he or she is elsewhere. And elsewhere that takes on an ever-increasing fascination, because it gives the reality of space a new connotation.» 
«Physical space in fact is emptied of significance, becomes less dense as thickness, as the dimension of virtual space is grafted on to it. This phenomenon has, however, the implication that more and more distance is created with the unknown. Today it is difficult to surrender oneself to sunknown lands because one can face them armed with a mobile, thus defended by the socialness of one's point of departure. As we said above, it is increasingly evident that between chosen and chance sociality the interest is much more on chosen, even if this is virtual. In fact, the more one is forced into mobility, that is, into travelling great distances towards the unknown, the more one has to stress relations with what is familiar.» This has taken the discussion of space, and the spatial context, to a discussion of agency and choices about which spaces to inhabit and which to avoid.

This analysis is not universally accepted. Some authors say mobiles attach people more strongly to existing social groups and contexts at the expense of joining or forming new ones; others, that they facilitate wide shallow affiliations at the expense of fewer deeper ones (Geser, 2004, McEwen, 2010). Whichever is actually the case, we are clearly seeing social proximity and social context changing from its established forms, being pulled and pushed and stretched differently.

She (Fortunati, 2002: 514) sees the underlying and unifying motivation for using mobile devices as somehow trying to wring more from both space and time: «The attempt is to enlarge the surface of space and the duration of time by means of communicative technologies. Space has widened out horizontally, lengthened out vertically, and at the same time is perceived as a background; while time is experienced in all its extensions and expanded in thickness. Space and time have thus become the new frontiers of increased social productivity.» This portrays users' temporal and spatial contexts as some kind of resource or raw material (spatiotemporal capital perhaps or space-time as a commodity) resonating with the capitalist injunction that «time is money) and connecting with the earlier Protestant valorisation of punctuality (Banks, 2006).

Time and space become more complex, they also become more confusing: «What space and time has the mobile found itself interacting with? With a space that was already transformed before, developing its technological aspect, informative, multicultural, mobile and relational, in short, becoming a complex space, not immediately easy to understand. The increasing difficulty in people's immediate and effective relation with space, which has become increasingly difficult to understand, has been an important element at the base of the spread and success of the mobile phone. This instrument, in fact, has enabled people to somehow attenuate their anxiety and bewilderment in the face of this new quality and dimension of space. The mobile phone is a device that enables people, when they perceive the surrounding environment as extraneous to them, to contact 
somebody of their intimate circle, that is, to activate the reassuring procedure of recognition. In other words, people react to the lack of informative immediacy of the place, strengthening communicative immediacy with their social networks by means of the mobile». (Fortunati, 2002:514) Therefore, temporal and spatial contexts are bewildering and unsettling.

\section{Different Spaces}

Mobile devices are reconfiguring the relationships between spaces, between public spaces and private ones, between public and private contexts, and the ways in which these are penetrated by mobile virtual spaces. This reconfiguration is accompanied by what goes on within those spaces. Cooper (2002:22) says that the private "is no longer conceivable as what goes on, discreetly, in the life of the individual away from the public domain, or as subsequently represented in individual consciousness» (Sheller \& Urry, 2003:1). «The use of these mobile sound technologies informs us about how users attempt to <inhabit the spaces within which they move. The use of these technologies appears to bind the disparate threads of much urban movement together, both sfillings the spaces in-betweens communication or meetings and structuring the spaces thus occupied» (Bull, 2005:344). More so, with the rise of noise-cancelling earphones, which further distance the local physical context.

This is part of a growing dislocation of time and place, in which «everything arrives without any need to depart» (Virilio, 2000:20). "Closer to what is far away than to what is just beside us, we are becoming progressively detached from ourselves» (Virilio, 2000:83). Owing to "the tendency to previsit locations, through one medium or another; to actually arrive somewhere is no longer surprising in the way that it was .... it is becoming replaced by prevision. Thus according to this logic, the mobile would be one more technique by which the world became unsurprising.» (Cooper, 2002:26) Another personal device, the in-car sat-nav, has a similar effect, that of previsiting places and locations. Other personal digital devices, such as the camcorder, the camera, allow us to recreate the past, to revisit places and locations, whilst augmented reality can supplement real places with imagined or imaginary ones. Moreover, «the instant availability of all types of information at any time or place means that there will be no need for physical motion» (Cooper, 2001:25); inertia or stagnation set in and the balance and proportions, the boundaries and the edges within the temporal context are transformed.

At an Eduserv conference in London in 2010, delegates were asked not to watch the presentations on the live stream on their own laptops, presentations taking place literally a few feet in front of them. Is this some preference for the virtual, the next stage in absent presence? 
There are a growing number of augmented reality applications available as retail downloads for smart-phones. These also add to the dilution of the immediate experience of the here-and-now. Examples include Layar, allowing developers to add layers onto the iPhone and Android phone's video, a (mash-up based on what the camera in the phone currently sees; Wikitude AR Travel Guide, which brings contextual Wikipedia information to what the camera currently sees; TAT Augmented ID, which uses the Flickr facial recognition technology to identify a person's face and overlay their image with their online profile and contact information and TwittARound, which takes an iPhone's camera and overlays live video of the world around it with tweets. Ever increasing exposure to CGI and Photoshop, for example, must mean that our relations with what we used to think of as 〈realitys are becoming ever more complex.

Augmented reality applications (Papagiannakis et al., 2008) and Google Maps with Street View both dilute the here-and-now; an ever-growing sense of surveillance and nervousness is implicit, too, as users become more known, visible and connected to their various contexts. Surveillance might be seen as a dilution of identity, watering down who one is, at the same time as digital identities become more complex. Identity becomes more complex and fluid as we acquire and discard digital identities and join and leave digital communities within which these digital identities have meaning making social contexts become more fluid.

\section{Interactive Travel through Contexts}

Elsewhere, authors (Germann-Molz, 2010) have commented on the «emergence of interactive travel, a mode of leisure travel that involves staying electronically connected while on the move». Characterised by travellers using mobile technologies «as a way of creating knowledge and negotiating on-the-road «knowhow. Leisure travellers are increasingly integrating mobile technologies such as laptop computers, wireless cards, MP3 players, GPS devices and mobile phones into their journeys in order to research and plan their trips, network with other travellers, share advice, and record, photograph and publish their experiences for the internet public. The result is a proliferation of online travel blogs, networked backpacker communities, mobile travel guides, hospitality networking sites, travel discussion boards, and the digital sharing of videos and photographs from travellers' journeys.» This is another way in which the real and virtual worlds, the real and virtual spatial contexts, are linked and interwoven; these travellers are «not only physically on the move, but are constantly moving amongst these overlapping virtual, imaginative, communicative and corporeal spaces of social interaction.» 


\section{Ragged and Uneven Contexts}

Of course, mobility and connectedness do not sweep away the boundaries of time and spaces. Nor, as we have seen, are the spatial and temporal contexts more homogeneous or isotropic. They have moorings and tethering of their own. Spaces are now defined by the availability of varieties of network coverage, by mains power sockets and by ambient lighting levels, without direct sunlight. Time is now measured by battery life; movement is restricted by cables, backing up and synching, in much the same way that, cars, the other symbol of modern Western mobility are tethered to servicing schedules, flat-tyres, traffic jams, car-parking spaces and filling stations. "Mobilities cannot be described without attention to the necessary spatial, infrastructural and institutional moorings that configure and enable mobilities ... There is no linear increase in fluidity without extensive systems of immobility ...» (Hannam et al., 2006)

\section{Individuals, their Identities and Communities}

Mobility and connection are also amongst the factors changing individuals and their identities, and the nature of communities. The rise of networked technologies has led to far more complex ideas about identity, both formally, in relation to ‘officialı network technologies, and informally, in relation to social networks. What constitutes the user, his/her self, as opposed to his/her context changes and blurs. Some authors describe personal mobile devices as becoming prosthetic; Pertierra (2005:27) says: «Unlike desktops and other immobile technologies, mobile phones more closely resemble tools or prosthetic devices as extensions of the body. They become extensions of the hand, allowing us to connect anytime, anywhere, with anybody. Bodies themselves become writing devices as phoneurs negotiate new urban spaces.» Other authors describe them as becoming embodied (for example, Rettie, 2005) or say: «From pacifier, to nipple, to digital umbilical cord, the mobile phone rapidly progressed to assume a vital place in the virtual biology of urban information societies of the late twentieth century» (Goggin, 2006). "At the final extreme, the mobile phone's connectivity might be completely subsumed into the body, and all other forms of communication become redundant email, web, phone calls, all can be delivered over the universal handheld» (Townsend, 2001:70). If context is a way of characterising the relationship between person and the environment, then what we are seeing this renegotiated in a variety of ways, in this case in the body and its tools. A different aspect of the embodiment of mobile phones was widely reported. «l'd rather» deadpans Philippa Grogan, 16, "give up, like, a kidney than my phone. How did you manage before? Carrier pigeons? Letters? Going round each other's houses on BIKES?» (Guardian 2010). So we see a much more fluid and fractured account of who we are, of our «selves», 
and likewise a more fluid and complex account of the physical environment and social communities that constitute our context.

\section{Knowledge, Knowing, Understanding and Learning}

Mobile devices affect the processes by which ideas, images, information and knowledge, and hence informal learning, are produced, stored, evaluated, valorised, distributed, delivered and consumed. They are now part of a system that allows everyone, including learners and potential learners, to generate and transmit content for learning, not just passively store and consume it, making mobile systems an integral part of the Web2.0 ideology that takes users from merely the Web's readers to its writers. The impact of mobility and connectedness on knowledge is to make it far more obviously relative, local, transient and partial. Knowledge is local in being local to a community, local in being location-specific, produced locally and consumed with defined communities, not necessarily geographically or spatially defined communities. The informational context is no longer fixed, monolithic and external.

In formal learning, we already see changed ideas about space and knowledge reflected in two emerging pedagogies, snavigationism> (Brown 2005) and «connectionism» (Siemens, 2004). These grow out of networked and mobile learning and shift the balance between ideas themselves and the relationships between them, emphasising a kind of knowledge space, a topology of points rather than a geography of bulky masses thus transforming the intellectual or cognitive context within which learners move. Connectivism explicitly assumes «knowledge is distributed across a network of connections, and therefore ... learning consists of the ability to construct and traverse those networks» (Downes, 2007). Therefore, we see changed perspectives on the relationships between knowledge and knower, or learning and the learner, with a far less straightforward relationship and boundary between each pair.

\section{The Future of Context}

In the words of a recent newspaper article (Bilton, 2010):

If you pull out your smartphone and click the button that says «locate me» on your mapping application, you will see a small dot appear in the middle of the screen.

That's you.

If you start walking down the street in any direction, the whole screen will move right along with you, no matter where you go.

This is a revolutionary change from the print-on-paper, where maps and locations are based around places and landmarks, not on you and your location. 
The newspaper article's title makes the point more succinctly: "Where the individual is front and center». Yes, in any consideration of context, the learner or user is (front and centre).

The early half of our argument endorses this idea and portrays the relationships between individual and environment as becoming increasingly richer and easier, as increasingly easy to exploit and deploy in support of existing, enhanced or reformed pedagogies, though recognising that that we, not our surroundings, are now the focus and the source. The second part of our argument, however, is that social changes mean that this user at ithe front and centres is no longer distinct and separate, no longer 'a small dot), more smeared and blurred in time, space, knowledge, community and identity, the boundary between 'self〉 and ‘other unsettled, dissolving and ill at ease. Perhaps we must also question whether the technologies of context are contributing to an enriched and augmented reality or just distracting our attention from a reality that technology is diluting, depleting and diminishing.

\section{References}

Agar, Jon (2003). Constant Touch: A Global History of the Mobile Phone. Cambridge: Icon.

Banks, Katherine (2006). L'Ordre du temps: I'invention de la ponctualité au XVle siècle. French Studies 2006 LX (1):97-98.

Bilton, Nick (2010). Where the individual is front and center. International Herald Tribune, Tuesday, September 14, 2010: 19.

Brown, Elizabeth (2010). Introduction to location-based mobile learning. In E. Brown (ed.), Education in the Wild: Contextual and Location-Based Mobile Learning in Action. Learning Sciences Research Institute, University of Nottingham.

Brown, Tom H. (2005). Beyond Constructivism: Exploring Future Learning Paradigms. Education Today, issue 2 of 2005, Aries Publishing Company: Thames, New Zealand.

Bull, Martin (2005). No Dead Air! The iPod and the Culture of Mobile Listening. Leisure Studies 24(4): 343-356.

Chen, Guanling; Kotz, David (2000). A Survey of Context-Aware Mobile Computing Research. Dartmouth Computer Science Technical Report TR2000-381.

Cook, John (2010). Mobile Learner Generated Contexts. Research on the Internalization of the World of Cultural Products. In Ben Bachmair (ed.) (2010). Medienbildung in neuen Kulturräumen: Die deutschsprachige und britische Diskussion, 113-126. Wiesbaden: VS Verlag für Sozialwissenschaften.

Cook, John; Pachler, Norbert; Bachmair, Ben (2010). Ubiquitous Mobility with Mobile Phones: A Cultural Ecology for Mobile Learning. 
Cooper, Geoff (2002). The Mutable World: Social Theory. In Barry Brown, Nicola Green and Richard Harper (eds), Wireless World: Social and Interactional Aspects of the Mobile World. London: Springer.

Dey, Anind K.; Abowd, Gregory D. (1999). Towards a Better Understanding of Context and Context-Awareness. GVU Technical Report GIT-GVU-99-22, College of Computing, Georgia Institute of Technology.

Dourish, Paul, (2004). What is we talk about when we talk about context. Personal and Ubiquitous Computing, 8(1), 19-30, February 2004. Available from http:// www.ics.uci.edu/ jpd/

Downes, Stephen (2007). What Connectivism Is. Available online at http://halfanhour.blogspot.com/2007/02/what-connectivism-is.html

Fortunati, Leopoldina (2002). The Mobile Phone: Towards New Categories and Social Relations. Information, Communication \& Society, 5(4): 513-528.

Germann-Molz, Jennie (2010). Connectivity, Collaboration, Search. In Monika Büscher, John Urry \& Katian Witchger (eds), Mobile Methods. Routledge: London.

Gergen, Kenneth J. (2002). The Challenge of Absent Presence. In J. E. Katz \& M. A. Aakhus (eds.), Perpetual Contact: Mobile Communication, Private Talk, Public Performance, 227-241. Cambridge University Press, Cambridge.

Goggin, Gerard (2006). Cell Phone Culture. Abingdon: Routledge.

Guardian (2010). The Guardian, July $16^{\text {th }}, 2010$ edition. Available online http:// www.guardian.co.uk/lifeandstyle/2010/jul/16/teenagers-mobiles-facebooksocial-networking

Hannam, Kevin; Sheller, Mimi; Urry, John (2006). Editorial: Mobilities, Immobilities and Moorings. Mobilities, 1(1): 1-22.

International Telecommunications Union (2004). Social and Human Considerations for a More Mobile World, Report from ITU/MIC Workshop on Shaping the Future Mobile Information Society, 26 February 2004, Seoul.

Ling, Rich (2004). The Mobile Connection - The Cell Phone's Impact on Society. San Francisco, CA: Morgan Kaufmann Publishers.

Ling, Rich (2008). New Tech, New Ties - How Mobile Communication Is Reshaping Social Cohesion. Cambridge, MA: MIT Press.

McEwen, Rhonda N. (2010). A World More Intimate: Exploring The Role of Mobile Phones in Maintaining and Extending Social Networks. Proceedings of Mobile Preconference, ICA Conference, Singapore: SIRC.

Nyíri, Kristof (2007). Mobile Studies: Paradigms and Perspectives. Vienna: Passagen Verlag: Hungarian Academy of Sciences.

Papagiannakis, George; Singh, Gurminder; Magnenat-Thalmann, Nadia (2008). A survey of mobile and wireless technologies for augmented reality systems. Journal of Computer Animation and Virtual Worlds, 19(1): 3-22. 
Pertierra, Raul (2005). Mobile Phones, Identity and Discursive Intimacy. An Interdisciplinary Journal on Humans in ICT Environments, 1(1).

Plant, Sadie (2001). On the Mobile - The Effects of Mobile Telephones on Individual and Social Life. Motorola.

Rettie, Ruth (2005). Presence and Embodiment in Mobile Phone Communication. Psychology Journal 3(1): 16-34.

Schilit, Bill; Theimer, Marvin (1994). Disseminating Active Map Information to Mobile Hosts. IEEE Netwk 8(5): 22-32.

Schmidt, Albrecht; Beigl, Michael; Gellesen, Hans-Werner (1998). There is more to context than location. International workshop on Interactive Applications of Mobile Computing, 893-901.

Sheller, Mimi; Urry, John (2003). Mobile Transformations of 〈Public〉 and ¿Private Lifes. Theory, Culture \& Society, Vol. 20: 107-125.

Siemens, George (2004). Connectivism: A Learning Theory for the Digital Age. International Journal of Instructional Technology and Distance Learning, January 2005, 2(1).

Siorpaes, Sven; Broll, Gregor; Paolucci, Massimo; Rukzio, Enrico; Hamard, John; Wagner, Matthias; Schmidt, Albrecht (2006). Mobile Interaction with the Internet of Things. Poster at $4^{\text {th }}$ International Conference on Pervasive Computing, Dublin, Ireland 2006. http://www.medien.informatik.unimuenchen.de/filead$\mathrm{min} / \mathrm{mimuc} /$ rukzio/Mobile_interaction_with_the_internet_of_things_final.pdf

Sørensen, Carsten; Mathiassen, Lars; Kakihara, Masao (2002). Mobile Services: Functional Diversity and Overload, presented at New Perspectives On $21^{\text {st }}$ Century Communications, May 24-25, 2002, Budapest, Hungary.

Stald, Gitte (2008). Mobile Identity: Youth, Identity, and Mobile Communication Media. In David Buckingham (ed.), Youth, Identity, and Digital Media, 143-164. The John D. and Catherine T. MacArthur Foundation Series on Digital Media and Learning. Cambridge, MA: The MIT Press.

Townsend, Anthony M, (2001). Mobile Communications in the Twenty-First Century City. In Barry Brown, Nicola Green and Richard Harper, Wireless World - Social and Interactional Aspects of the Mobile World. London: Springer.

Traxler, John (2010a). Students and Mobile Devices. Research in Learning Technology, 18(2): 149-160.

Traxler, John (2010b). Sustaining Mobile Learning and Its Institutions. International Journal of Mobile and Blended Learning, 2(4): 129-138.

Virilio, Paul (2000). Polar Inertia. London: Sage.

Wang, Yuan-Kai (2004). Context-Awareness and Adaptation in Mobile Learning. Proceedings of International workshop on Mobile Technologies in Education, (WMTE), 154-158. 